\title{
Non-separability of the Lipschitz distance
}

\author{
Kohei Suzuki and Yohei Yamazaki
}

\section{Abstract}

Let $X$ be a compact metric space and $\mathcal{M}_{X}$ be the set of isometry classes of compact metric spaces $Y$ such that the Lipschitz distance $d_{L}(X, Y)$ is finite. We show that $\left(\mathcal{M}_{X}, d_{L}\right)$ is not separable when $X$ is a closed interval, or an infinite union of shrinking closed intervals.

Keywords: Lipschitz convergence; compact metric spaces; separability

\section{Introduction}

For compact metric spaces $\left(X, d_{X}\right)$ and $\left(Y, d_{Y}\right)$, the Lipschitz distance $d_{L}(X, Y)$ is defined to be the infimum of $\epsilon \geq 0$ such that an $\epsilon$-isometry $f: X \rightarrow Y$ exists. Here a bi-Lipschitz homeomorphism $f: X \rightarrow Y$ is called an $\epsilon$-isometry if

$$
|\log \operatorname{dil}(f)|+\left|\log \operatorname{dil}\left(f^{-1}\right)\right| \leq \epsilon,
$$

where $\operatorname{dil}(f)$ denotes the smallest Lipschitz constant of $f$, called the dilation off:

$$
\operatorname{dil}(f)=\sup _{\substack{x, y \in X \\ x \neq y}} \frac{d_{Y}(f(x), f(y))}{d_{X}(x, y)} .
$$

Let $\mathcal{M}$ be the set of isometry classes of compact metric spaces. It is well-known that $\left(\mathcal{M}, d_{L}\right)$ is a complete metric space. See, e.g., ([4] Appendix A) for the proof of the completeness and see, e.g., [1,2] for details of the Lipschitz distance.

Then the following question arises:

$(Q)$ : Is the metric space $\left(\mathcal{M}, d_{L}\right)$ separable?

The answer is no, which can be seen easily by the following facts:

(a) if $d_{L}(X, Y)<\infty$, the Hausdorff dimensions of $X$ and $Y$ must coincide;

(b) for any non-negative real number $d$, there is a compact metric space $X$ whose Hausdorff dimension is equal to $d$.

See, e.g., ([1] Proposition 1.7.19) for (a) and [3] for (b).

*Correspondence: kohei0604@math.kyoto-u.ac.jp

Department of Mathematics, Faculty of Science, Kyoto University, Oiwake-Cho,

Kitashirakawa Sakyo-Ku, Kyoto-Shi, Kyoto, 606-8502, Japan
The fact (b) indicates that $\left(\mathcal{M}, d_{L}\right)$ is too big to be separable. Then we change the question $(\mathrm{Q})$ to the following more reasonable one (Q'): For a compact metric space $X$, let $\mathcal{M}_{X}$ be the set of isometry classes of compact metric spaces $Y$ such that $d_{L}(X, Y)<\infty$. Any elements of $\mathcal{M}_{X}$ have a common Hausdorff dimension by (a). Then the following question arises:

$\left(Q^{\prime}\right):$ Is the metric space $\left(\mathcal{M}_{X}, d_{L}\right)$ separable?

The main results of this paper give the negative answer for this question for several $X$. To be more precise, we give two examples for $X$ such that $\left(\mathcal{M}_{X}, d_{L}\right)$ is not separable:

(i) Infinite unions of shrinking closed intervals with zero

$$
\{0\} \cup \bigcup_{n=1}^{\infty}\left[\frac{1}{2^{n}}, \frac{1}{2^{n}}+\frac{1}{2^{n+1}}\right] ;
$$

(ii) Closed interval $[0,1]$.

We would like to stress that $\left(\mathcal{M}_{X}, d_{L}\right)$ becomes nonseparable even when $X$ are the above elementary cases. We note that the non-separability of the first example follows from the non-separability of the second example. The first example, however, is easier to show the non-separability than the second example.

The present paper is organized as follows: In the first section, we show that the set of isometry classes of the infinite unions of shrinking closed intervals with zero is not separable. In the second section, we show that the set of isometry classes of the closed interval is not separable.

\section{Springer}




\section{The first example}

Let $\mathbb{Z}_{>0}=\left\{n \in \mathbb{Z}_{>0}: n>0\right\}$ denote the set of positive integers. For $n, m \in \mathbb{Z}_{>0}$, let $I(n, m)$ be an interval in $\mathbb{R}$ defined as follows:

$$
I(n, m)=\left[\frac{1}{2^{n}}, \frac{1}{2^{n}}+\frac{1}{2^{n+m}}\right] .
$$

For each $u=\left(u_{n}\right)_{n \in \mathbb{Z}_{>0}} \in\{1,2\}^{\mathbb{Z}_{>0}}$, we define the following subset in $\mathbb{R}$ :

$$
X_{u}=\{0\} \cup \bigcup_{n=1}^{\infty} I\left(n, u_{n}\right) .
$$

We equip $X_{u}$ with the usual Euclidean metric in $\mathbb{R}$ :

$$
d(x, y)=|x-y|, \quad x, y \in X_{u} .
$$

Then it is easy to check that $\left(X_{u}, d\right)$ is a compact metric space.

Let $\mathbf{1}=(1,1,1, \ldots) \in\{1,2\}^{\mathbb{Z}_{>0}}$ denote the element in $\{1,2\}^{\mathbb{Z}_{>0}}$ such that all components are equal to one. Let $X_{1}$ be the set defined in (1) for the element 1. Let $\mathcal{M}_{X_{1}}$ denote the set of isometry classes of compact metric spaces $X$ whose Lipschitz distances from $X_{1}$ are finite, that is, $d_{L}\left(X, X_{1}\right)<\infty$. Then we have the following result:

Theorem 1. $\left(\mathcal{M}_{X_{1}}, d_{L}\right)$ is not separable.

Proof 1. It is enough to find a certain discrete subset $\mathbb{X} \subset$ $\mathcal{M}_{X_{1}}$ with the continuous cardinality. We introduce a subset $\mathbb{X} \subset \mathcal{M}$, which is the set of isometry classes of all $X_{u}$ for $u \in \mathbb{Z}_{>0}$ :

$$
\mathbb{X}=\left\{\left(X_{u}, d\right): u \in\{1,2\}^{\mathbb{Z}}\right\} \text { /isometry }
$$

It is clear that the cardinality of $\mathbb{X}$ is continuum. We show that $\mathbb{X} \subset \mathcal{M}_{X_{1}}$ and $\mathbb{X}$ is discrete (i.e., every point in $\mathbb{X}$ is isolated).

We first show that $\mathbb{X} \subset \mathcal{M}_{X_{1}}$. For $u=\left(u_{n}\right)_{n \in \mathbb{Z}_{>0}} \in$ $\{1,2\}^{\mathbb{Z}_{>0}}$ and $v=\left(v_{n}\right)_{n \in \mathbb{Z}_{>0}} \in\{1,2\}^{\mathbb{Z}_{>0}}$, let $f_{u, v}$ be $a$ function from $X_{u}$ to $X_{v}$ defined by

$$
f_{u, v}(x)=\left\{\begin{array}{l}
0 \quad(x=0), \\
\frac{2^{u_{n}}}{2^{v_{n}}}\left(x-\frac{1}{2^{n}}\right)+\frac{1}{2^{n}} \quad\left(x \in I\left(n, u_{n}\right)\right) .
\end{array}\right.
$$

Then $f_{u, v}$ is a bi-Lipschitz continuous function from $X_{u}$ to $X_{v}$ and for $x, y \in X_{u}$,

$$
\frac{1}{2}|x-y| \leq\left|f_{u, v}(x)-f_{u, v}(y)\right| \leq 2|x-y| .
$$

Therefore the Lipschitz distance between $X_{u}$ and $X_{v}$ is bounded by

$$
d_{L}\left(X_{u}, X_{v}\right) \leq 2 \log 2 \text { for any } u, v \in \mathbb{Z}_{>0} .
$$

Thus we have that $\mathbb{X} \subset \mathcal{M}_{X_{1}}$. Second we show that $\mathbb{X}$ is discrete:

Lemma 1. Let $X_{u}, X_{v} \in \mathbb{X}$. If $d_{L}\left(X_{u}, X_{v}\right)<\log 2$, then $u=v$.

Proof 2. Let $u=\left(u_{n}\right)_{n \in \mathbb{Z}_{>0}} \in\{1,2\}^{\mathbb{Z}_{>0}}$ and $v=$ $\left(v_{n}\right)_{n \in \mathbb{Z}_{>0}} \in\{1,2\}^{\mathbb{Z}_{>0}}$. We show that $u_{n}=v_{n}$ for all $n \in$ $\mathbb{Z}_{>0}$. By the assumption $d_{L}\left(X_{u}, X_{v}\right)<\log 2$, there exists a bi-Lipschitz function $f: X_{u} \rightarrow X_{v}$ such that

$$
|\log \operatorname{dil}(f)|+\left|\log \operatorname{dil}\left(f^{-1}\right)\right|<\log 2 .
$$

Since $f$ is homeomorphic, any intervals must be mapped to intervals by $f$. That is, there exists a bijection $P: \mathbb{Z}_{>0} \rightarrow$ $\mathbb{Z}_{>0}$ as $n \mapsto P(n)$ such that

$$
f\left(I\left(n, u_{n}\right)\right)=I\left(P(n), v_{P(n)}\right) .
$$

To show $u_{n}=v_{n}$ for all $n \in \mathbb{Z}_{>0}$, we have two steps:

(i) $n+u_{n}=P(n)+v_{P(n)}$;

(ii) $P(n)=n$.

We start to show (i) by contradiction. Assume there exists $n_{0} \in \mathbb{Z}_{>0}$ such that $n_{0}+u_{n_{0}} \neq P\left(n_{0}\right)+v_{P\left(n_{0}\right)}$. Since $\left.f\right|_{I\left(n_{0}, u_{n_{0}}\right)}$ is homeomorphic, the endpoints of $I\left(n_{0}, u_{n_{0}}\right)$ must be mapped to the endpoints of $I\left(P\left(n_{0}\right), v_{P\left(n_{0}\right)}\right)$ by $\left.f\right|_{I\left(n_{0}, u_{n_{0}}\right)}$. Therefore

$$
\left|f\left(\frac{1}{2^{n_{0}}}\right)-f\left(\frac{1}{2^{n_{0}}}+\frac{1}{2^{n_{0}+u_{n_{0}}}}\right)\right|=\frac{1}{2^{P\left(n_{0}\right)+v_{P\left(n_{0}\right)}}} .
$$

Thus the dilation off is at least bigger than

$$
\begin{aligned}
\operatorname{dil}(f) & \geq \frac{\left|f\left(1 / 2^{n_{0}}\right)-f\left(1 / 2^{n_{0}}+1 / 2^{n_{0}+u_{n_{0}}}\right)\right|}{\left|1 / 2^{n_{0}}-\left(1 / 2^{n_{0}}+1 / 2^{n_{0}+u_{n_{0}}}\right)\right|} \\
& =\frac{1}{2^{P\left(n_{0}\right)+v_{P\left(n_{0}\right)}-\left(n_{0}+u_{n_{0}}\right)}} .
\end{aligned}
$$

By the assumption of $n_{0}+u_{n_{0}} \neq P\left(n_{0}\right)+v_{P\left(n_{0}\right)}$, we have that $\operatorname{dil}(f) \geq 2$ ordil $\left(f^{-1}\right) \geq 2$. This implies

$$
|\log \operatorname{dil}(f)| \geq \log 2 \quad \text { or } \quad\left|\log \operatorname{dil}\left(f^{-1}\right)\right| \geq \log 2 .
$$

This contradicts the inequality (2). Hence we have $n+$ $u_{n}=P(n)+v_{P(n)}$ for all $n \in \mathbb{Z}_{>0}$.

We start to show (ii) by contradiction. Assume there exists $n_{0} \in \mathbb{Z}_{>0}$ such that $P\left(n_{0}\right) \neq n_{0}$. Let us define

$$
n_{*}=\min \left\{n \in \mathbb{Z}_{>0} \mid P(n) \neq n\right\} .
$$

Then $P\left(n_{*}\right)>n_{*}$ by definition. Since we know that $n+$ $u_{n}=P(n)+v_{P(n)}$ by the first step $(i)$, and that $u_{n}$ and $v_{P(n)}$ are in $\{1,2\}$, thus the possibility of values of $P(n)$ is that $P(n)=n-1, n$ or $n+1$. This implies that $P\left(n_{*}\right)=n_{*}+1$, $P\left(n_{*}+1\right)=n_{*}$ and $P\left(n_{*}+2\right)=n_{*}+2$, or $n_{*}+3$. Since the endpoints of intervals must be mapped to the endpoints of 
intervals by $f$, the possibility of values of $f\left(1 / 2^{n_{*}+1}\right)$ and $f\left(1 / 2^{n_{*}+2}\right)$ is

$$
f\left(\frac{1}{2^{n_{*}+1}}\right)=\frac{1}{2^{n_{*}}}, \quad \text { or } \quad \frac{1}{2^{n_{*}}}+\frac{1}{2^{n_{*}+v_{n_{*}}}},
$$

and

$$
\begin{gathered}
f\left(\frac{1}{2^{n_{*}+2}}\right)=\frac{1}{2^{n_{*}+2}}, \frac{1}{2^{n_{*}+2}}+\frac{1}{2^{n_{*}+2+v_{\left(n_{*}+2\right)}}}, \frac{1}{2^{n_{*}+3}}, \\
\text { or } \frac{1}{2^{n_{*}+3}}+\frac{1}{2^{n_{*}+3+v_{\left(n_{*}+3\right)}}} .
\end{gathered}
$$

Thus, by noting $v_{P\left(n_{*}+2\right)} \in\{1,2\}$, we have the following estimate:

$$
\begin{aligned}
& \left|f\left(\frac{1}{2^{n_{*}+1}}\right)-f\left(\frac{1}{2^{n_{*}+2}}\right)\right| \\
& \quad \geq\left|\frac{1}{2^{n_{*}}}-\left(\frac{1}{2^{n_{*}+2}}+\frac{1}{2^{n_{*}+2+v_{P\left(n_{*}+2\right)}}}\right)\right| \\
& \quad \geq \frac{5}{2} \frac{1}{2^{n_{*}+2}} .
\end{aligned}
$$

This shows $|\log \operatorname{dil}(f)| \geq \log (5 / 2)$ and contradicts the inequality (2). Hence we have $P(n)=n$ for all $n \in \mathbb{Z}_{>0}$.

By the above two steps, we have that $u_{n}=v_{n}$ for all $n \in$ $\mathbb{Z}_{>0}$, and we have completed the proof of Lemma 1.

We resume the proof of Theorem 1.

Proof of Theorem 1. By using Lemma 1, we know that $\left(\mathbb{X}, d_{L}\right)$ is discrete. Since the cardinality of $\mathbb{X}$ is continuum and $\mathbb{X} \subset \mathcal{M}_{X_{1}}$, we have that $\left(\mathcal{M}_{X_{1}}, d_{L}\right)$ is not separable. We have completed the proof.

\section{The second example}

In this section, we show the non-separability of $\mathcal{M}_{[0,1]}$ :

Theorem 2. The metric space $\left(\mathcal{M}_{[0,1]}, d_{L}\right)$ is not separable.

Proof. It is enough to find a certain discrete subset $\mathbb{Y} \subset$ $\mathcal{M}_{[0,1]}$ with the continuous cardinality.

Define two subsets, flat parts $J(n, 0)$, and pulse parts $J(n, 1)$ in $\mathbb{R}^{2}$ :

- Flat part: for $n \in \mathbb{Z}_{>0}$,

$$
J(n, 0)=\left[\frac{1}{2^{n}}, \frac{1}{2^{n-1}}\right] \times\{0\},
$$

- Pulse part: for $n \in \mathbb{Z}_{>0}$,

$$
\begin{aligned}
J(n, 1) & =\left[\frac{3}{2^{n+1}}, \frac{1}{2^{n-1}}\right] \times\{0\} \\
& \cup\left\{\left(x, \frac{3}{2^{n+1}}-x\right): \frac{5}{2^{n+2}} \leq x \leq \frac{3}{2^{n+1}}\right\} \\
& \cup\left\{\left(x, x-\frac{1}{2^{n}}\right): \frac{1}{2^{n}} \leq x \leq \frac{5}{2^{n+2}}\right\} .
\end{aligned}
$$

See the Figure 1.

For each $u=\left(u_{n}\right)_{n \in \mathbb{Z}_{>0}} \in\{0,1\}^{\mathbb{Z}_{>0}}$, let $Y_{u}$ be a subset in $\mathbb{R}^{2}$ as an infinite union of flat parts and pulse parts with the origin:

$$
Y_{u}=\{(0,0)\} \cup \bigcup_{n=1}^{\infty} J\left(n, u_{n}\right) \subset \mathbb{R}^{2} .
$$

See the Figure 2.

We equip $Y_{u}$ with the usual Euclidean distance in $\mathbb{R}^{2}$ :

$$
d\left(\left(x_{1}, x_{2}\right),\left(y_{1}, y_{2}\right)\right)=\left(\left(x_{1}-y_{1}\right)^{2}+\left(x_{2}-y_{2}\right)^{2}\right)^{1 / 2} \text {. }
$$

It is easy to check that $\left(Y_{u}, d\right)$ is a compact metric space. Let $\mathbb{Y}$ be the set of isometry classes of $Y_{u}$ for all $u \in$ $\{0,1\}^{\mathbb{Z}_{>0}}$ :

$$
\mathbb{Y}=\left\{Y_{u}: u \in\{0,1\}^{\mathbb{Z}_{>0}}\right\} / \text { isometry. }
$$

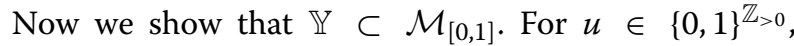
let $f_{u}$ be the projection from $Y_{u}$ to $[0,1]$ such that $x=$ $\left(x_{1}, x_{2}\right) \mapsto x_{1}$. Then it is easy to see that $f_{u}$ is bi-Lipschitz continuous and, for $x, y \in Y_{u}$,

$$
\frac{1}{\sqrt{2}} d(x, y) \leq\left|f_{u}(x)-f_{u}(y)\right| \leq d(x, y) .
$$

Therefore the Lipschitz distance between $[0,1]$ and $Y_{u}$ is bounded by

$$
d_{L}\left([0,1], Y_{u}\right) \leq \frac{1}{2} \log 2 \quad \forall u \in\{0,1\}^{\mathbb{Z}_{>0}} .
$$

Thus we have $\mathbb{Y} \subset \mathcal{M}_{[0,1]}$.

Now we show that $\mathbb{Y}$ is discrete:

Lemma 2. Let $Y_{u}, Y_{v} \in \mathbb{Y}$. If

$$
d_{L}\left(Y_{u}, Y_{v}\right)<\frac{\log (\sqrt{2}+1)-\log \sqrt{5}}{2},
$$

then $u=v$.

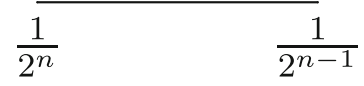

Figure 1 The left is $J(n, 0)$ and the right is $J(n, 1)$.

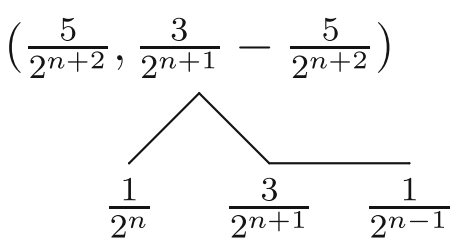




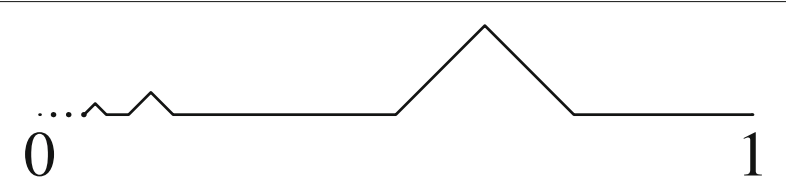

Figure 2 A picture of $Y_{u}$.

Proof 3. Let $u=\left(u_{n}\right)_{n \in \mathbb{Z}_{>0}} \in\{1,2\}^{\mathbb{Z}_{>0}}$ and $v=$ $\left(v_{n}\right)_{n \in \mathbb{Z}_{>0}} \in\{1,2\}^{\mathbb{Z}_{>0}}$. We show that $u_{n}=v_{n}$ for all $n \in$ $\mathbb{Z}_{>0}$. By the assumption, there exists a bi-Lipschitz function $f$ from $Y_{u}$ to $Y_{v}$ such that

$|\log \operatorname{dil}(f)|+\left|\log \operatorname{dil}\left(f^{-1}\right)\right|<\frac{\log (\sqrt{2}+1)-\log \sqrt{5}}{2}$.

Let us define a subset in $\mathbb{Z}_{>0}$ as follows:

$$
P_{u}=\left\{n \in \mathbb{Z}_{>0}: u_{n}=1\right\} .
$$

Without loss of generality, we may assume that $P_{u}$ is not empty. That is, $Y_{u}$ has at least one pulse. The pulse part $J\left(n, u_{n}\right)$ of $Y_{u}$ for $n \in P_{u}$ is called n-pulse of $Y_{u}$. We note that, by the definition of the pulse parts, the peak of the $n$-pulse is attained at $5 / 2^{n+2}$ in $x$-axis.

It is enough for the desired result to show that $P_{u}=P_{v}$. We show that there is a bijection $F: P_{u} \rightarrow P_{v}$ such that $F(n)=n$. To show this, we have the following three steps:

(i) The first step: for $n \in P_{u}$,

$$
\begin{aligned}
f_{v} \circ f \circ f_{u}^{-1}\left(\frac{5}{2^{n+2}}\right) & \in\left\{\frac{5}{2^{m+2}}: m \in \mathbb{Z}_{>0}\right\} \\
& \cup\left\{\frac{3}{2^{m+1}}: m \in \mathbb{Z}_{>0}\right\} \\
& \cup\left\{\frac{1}{2^{m}}: m \in \mathbb{Z}_{>0}\right\} .
\end{aligned}
$$

(ii) The second step: for $n \in P_{u}$,

$$
\begin{aligned}
f_{v} \circ f \circ f_{u}^{-1}\left(\frac{5}{2^{n+2}}\right) & \notin\left\{\frac{3}{2^{m+1}}: m \in \mathbb{Z}_{>0}\right\} \\
& \cup\left\{\frac{1}{2^{m}}: m \in \mathbb{Z}_{>0}\right\} .
\end{aligned}
$$

(iii) The third step: for $n \in P_{u}$,

$$
f_{v} \circ f \circ f_{u}^{-1}\left(\frac{5}{2^{n+2}}\right)=\frac{5}{2^{n+2}} \quad \text { and } \quad v_{n}=1 .
$$

In fact, if we show the above three statements, each maximizers $5 / 2^{n+2}$ of $n$-pulses of $Y_{u}$ are mapped to the maximizers $5 / 2^{n+2}$ of $n$-pulses of $Y_{v}$ by $f_{v} \circ f \circ f_{u}^{-1}$. This correspondence of n-pulses defines the map $F: P_{u} \rightarrow P_{v}$ such that $F(n)=n$.

The proof of the all three steps (i)-(iii) are governed by the same scheme:

(A) Assume that the statements do not hold (proof by contradiction);

(B) Estimate lower bounds of the dilations off and $f^{-1}$;

(C) The lower bounds obtained in (B) contradict the inequality (5).

We start to show the first step (i). Since $f$ is homeomorphic, the maximizer $5 / 2^{n+2}$ of the pulse cannot be mapped to the endpoints of $[0,1]$ by $f_{v} \circ f \circ f_{u}^{-1}$. Assume that, for some $n \in P_{u}$,

$$
\begin{aligned}
f_{v} \circ f \circ f_{u}^{-1}\left(\frac{5}{2^{n+2}}\right) & \notin\left\{\frac{5}{2^{m+2}}: m \in \mathbb{Z}_{>0}\right\} \\
& \cup\left\{\frac{3}{2^{m+1}}: m \in \mathbb{Z}_{>0}\right\} \\
& \cup\left\{\frac{1}{2^{m}}: m \in \mathbb{Z}_{>0}\right\},
\end{aligned}
$$

and prove (i) by contradiction. By the continuity of $f$, there exists $0<\delta<1 / 2^{n+3}$ such that, for any $x \in\left[5 / 2^{n+2}-\right.$ $\left.\delta, 5 / 2^{n+2}+\delta\right]$, we have

$$
\begin{aligned}
f_{v} \circ f \circ f_{u}^{-1}(x) & \notin\left\{\frac{5}{2^{m+2}}: m \in \mathbb{Z}_{>0}\right\} \\
& \cup\left\{\frac{3}{2^{m+1}}: m \in \mathbb{Z}_{>0}\right\} \\
& \cup\left\{\frac{1}{2^{m}}: m \in \mathbb{Z}_{>0}\right\} .
\end{aligned}
$$

Therefore we have

$$
\begin{aligned}
d( & \left.f \circ f_{u}^{-1}\left(\frac{5}{2^{n+2}}-\delta\right), f \circ f_{u}^{-1}\left(\frac{5}{2^{n+2}}+\delta\right)\right) \\
= & d\left(f \circ f_{u}^{-1}\left(\frac{5}{2^{n+2}}-\delta\right), f \circ f_{u}^{-1}\left(\frac{5}{2^{n+2}}\right)\right) \\
& +d\left(f \circ f_{u}^{-1}\left(\frac{5}{2^{n+2}}\right), f \circ f_{u}^{-1}\left(\frac{5}{2^{n+2}}+\delta\right)\right) .
\end{aligned}
$$

Here we use the fact that the three points $f \circ f_{u}^{-1}\left(5 / 2^{n+2}-\right.$ $\delta), f \circ f_{u}^{-1}\left(5 / 2^{n+2}\right)$ and $f \circ f_{u}^{-1}\left(5 / 2^{n+2}+\delta\right)$ are on the 
same line. By using the inequality (4), the dilation of $f$ is estimated as follows:

$$
\begin{aligned}
\operatorname{dil}(f) \geq & \frac{d\left(f \circ f_{u}^{-1}\left(\frac{5}{2^{n+2}}-\delta\right), f \circ f_{u}^{-1}\left(\frac{5}{2^{n+2}}+\delta\right)\right)}{d\left(f_{u}^{-1}\left(\frac{5}{2^{n+2}}-\delta\right), f_{u}^{-1}\left(\frac{5}{2^{n+2}}+\delta\right)\right)} \\
= & \frac{d\left(f \circ f_{u}^{-1}\left(\frac{5}{2^{n+2}}-\delta\right), f \circ f_{u}^{-1}\left(\frac{5}{2^{n+2}}\right)\right)}{2 \delta} \\
& +\frac{d\left(f \circ f_{u}^{-1}\left(\frac{5}{2^{n+2}}\right), f \circ f_{u}^{-1}\left(\frac{5}{2^{n+2}}+\delta\right)\right)}{2 \delta} \\
\geq & \frac{d\left(f_{u}^{-1}\left(\frac{5}{2^{n+2}}-\delta\right), f_{u}^{-1}\left(\frac{5}{2^{n+2}}\right)\right)}{2 \delta d i l\left(f^{-1}\right)} \\
= & \frac{d\left(f_{u}^{-1}\left(\frac{5}{2^{n+2}}\right), f_{u}^{-1}\left(\frac{5}{2^{n+2}}+\delta\right)\right)}{2 \delta d i l\left(f^{-1}\right)}
\end{aligned}
$$

In the above last line, we just calculated the distance following the Euclidean distance (3) in the n-pulse $J(n, 1)$. This implies that $\operatorname{dil}(f) \geq 2^{\frac{1}{4}}$, or $\operatorname{dil}\left(f^{-1}\right) \geq 2^{\frac{1}{4}}$. Thus we have

$$
d_{L}\left(Y_{u}, Y_{v}\right) \geq \frac{\log 2}{4}
$$

This contradicts the inequality (5). Therefore we have, for any $n \in P_{u}$,

$$
\begin{aligned}
f_{v} \circ f \circ f_{u}^{-1}\left(\frac{5}{2^{n+2}}\right) & \in\left\{\frac{5}{2^{m+2}}: m \in \mathbb{Z}_{>0}\right\} \\
& \cup\left\{\frac{3}{2^{m+1}}: m \in \mathbb{Z}_{>0}\right\} \\
& \cup\left\{\frac{1}{2^{m}}: m \in \mathbb{Z}_{>0}\right\} .
\end{aligned}
$$

We start to show the second step (ii) by contradiction. Assume that, for some $n \in P_{u}$,

$$
f_{v} \circ f \circ f_{u}^{-1}\left(\frac{5}{2^{n+2}}\right) \in\left\{\frac{1}{2^{m}}: m \in \mathbb{Z}_{>0}\right\} .
$$

Then there exists $n_{1} \in \mathbb{Z}_{>0}$ such that

$$
f_{v} \circ f \circ f_{u}^{-1}\left(\frac{5}{2^{n+2}}\right)=\frac{1}{2^{n_{1}}} .
$$

By the same argument as the first step (i), we can obtain $v_{n_{1}}=1$, that is, the $n_{1}$-pulse exists in $Y_{v}$. By the continuity off, there exists $0<\delta<1 / 2^{n_{1}+3}$ such that

$$
\begin{aligned}
f_{u} \circ f^{-1} \circ f_{v}^{-1}\left(\left[\frac{1}{2^{n_{1}}}-\delta, \frac{1}{2^{n_{1}}}\right)\right) & \subset\left(\frac{5}{2^{n+2}}-\frac{1}{2^{n+3}}, \frac{5}{2^{n+2}}\right) \\
& \cup\left(\frac{5}{2^{n+2}}, \frac{5}{2^{n+2}}+\frac{1}{2^{n+3}}\right),
\end{aligned}
$$

and

$$
\begin{aligned}
f_{u} \circ f^{-1} \circ f_{v}^{-1}\left(\left(\frac{1}{2^{n_{1}}}, \frac{1}{2^{n_{1}}}+\delta\right]\right) & \subset\left(\frac{5}{2^{n+2}}-\frac{1}{2^{n+3}}, \frac{5}{2^{n+2}}\right) \\
& \cup\left(\frac{5}{2^{n+2}}, \frac{5}{2^{n+2}}+\frac{1}{2^{n+3}}\right) .
\end{aligned}
$$

Noting the definition of $\left(Y_{v}, d\right)$, for $x \in\left[\frac{1}{2^{n_{1}}}-\delta, \frac{1}{2^{n_{1}}}\right)$ and $y \in\left(\frac{1}{2^{n_{1}}}, \frac{1}{2^{n_{1}}}+\delta\right]$, we have

$$
\begin{aligned}
d\left(f_{v}^{-1}(x), f_{v}^{-1}(y)\right) & =\left(|x-y|^{2}+\left|y-\frac{1}{2^{n_{1}}}\right|^{2}\right)^{1 / 2}, \\
d\left(f_{v}^{-1}(x), f_{v}^{-1}\left(\frac{1}{2^{n_{1}}}\right)\right) & =\left|x-\frac{1}{2^{n_{1}}}\right| \\
d\left(f_{v}^{-1}(y), f_{v}^{-1}\left(\frac{1}{2^{n_{1}}}\right)\right) & =\sqrt{2}\left|y-\frac{1}{2^{n_{1}}}\right| .
\end{aligned}
$$

Since $|x-y|=\left|x-2^{-n_{1}}\right|+\left|y-2^{-n_{1}}\right|$, we have the following inequality:

$$
\begin{aligned}
d( & \left.f_{v}^{-1}(x), f_{v}^{-1}\left(\frac{1}{2^{n_{1}}}\right)\right)+d\left(f_{v}^{-1}(y), f_{v}^{-1}\left(\frac{1}{2^{n_{1}}}\right)\right) \\
= & \left(\left|x-2^{-n_{1}}\right|^{2}+2 \sqrt{2}\left|x-2^{-n_{1}}\right|\left|y-2^{-n_{1}}\right|\right. \\
& \left.+2\left|y-2^{-n_{1}}\right|^{2}\right)^{1 / 2} \\
\leq & \left(\sqrt{2}\left|x-2^{-n_{1}}\right|^{2}+2 \sqrt{2}\left|x-2^{-n_{1}}\right|\left|y-2^{-n_{1}}\right|\right. \\
& \left.+2 \sqrt{2}\left|y-2^{-n_{1}}\right|^{2}\right)^{1 / 2} \\
= & 2^{\frac{1}{4}} d\left(f_{v}^{-1}(x), f_{v}^{-1}(y)\right) .
\end{aligned}
$$

On the other hand, there exist $x_{0} \in\left[\frac{1}{2^{n_{1}}}-\delta, \frac{1}{2^{n_{1}}}\right)$ and $y_{0} \in\left(\frac{1}{2^{n_{1}}}, \frac{1}{2^{n_{1}}}+\delta\right]$ such that

$$
\begin{aligned}
& d\left(f^{-1} \circ f_{v}^{-1}\left(x_{0}\right), f_{u}^{-1}\left(\frac{5}{2^{n+2}}\right)\right) \\
& \quad=d\left(f^{-1} \circ f_{v}^{-1}\left(y_{0}\right), f_{u}^{-1}\left(\frac{5}{2^{n+2}}\right)\right) .
\end{aligned}
$$

Thus the triangle determined by the three vertices $f \circ$ $f_{v}^{-1}\left(x_{0}\right), f \circ f_{v}^{-1}\left(y_{0}\right)$ and $f_{u}^{-1}\left(5 / 2^{n+2}\right)$ is an isosceles right triangle, and we can calculate

$$
\begin{aligned}
d\left(f^{-1} \circ f_{v}^{-1}\left(x_{0}\right), f^{-1} \circ f_{v}^{-1}\left(y_{0}\right)\right) & \\
= & \frac{1}{\sqrt{2}} d\left(f^{-1} \circ f_{v}^{-1}\left(x_{0}\right), f_{u}^{-1}\left(\frac{5}{2^{n+2}}\right)\right) \\
& +\frac{1}{\sqrt{2}} d\left(f^{-1} \circ f_{v}^{-1}\left(y_{0}\right), f_{u}^{-1}\left(\frac{5}{2^{n+2}}\right)\right) .
\end{aligned}
$$


By (6), (7) and (8), we have a bound for the dilation off:

$$
\begin{aligned}
\frac{1}{\operatorname{dil}(f)} \leq & \frac{d\left(f^{-1} \circ f_{v}^{-1}\left(x_{0}\right), f^{-1} \circ f_{v}^{-1}\left(y_{0}\right)\right)}{d\left(f_{v}^{-1}\left(x_{0}\right), f_{v}^{-1}\left(y_{0}\right)\right)} \\
= & \frac{d\left(f^{-1} \circ f_{v}^{-1}\left(x_{0}\right), f_{u}^{-1}\left(\frac{5}{2^{n+2}}\right)\right)}{\sqrt{2} d\left(f_{v}^{-1}\left(x_{0}\right), f_{v}^{-1}\left(y_{0}\right)\right)} \\
& +\frac{d\left(f^{-1} \circ f_{v}^{-1}\left(y_{0}\right), f_{u}^{-1}\left(\frac{5}{2^{n+2}}\right)\right)}{\sqrt{2} d\left(f_{v}^{-1}\left(x_{0}\right), f_{v}^{-1}\left(y_{0}\right)\right)} \\
\leq & \frac{\operatorname{dil}\left(f^{-1}\right) d\left(f_{v}^{-1}\left(x_{0}\right), f_{v}^{-1}\left(\frac{1}{2^{n_{1}}}\right)\right)}{\sqrt{2} d\left(f_{v}^{-1}\left(x_{0}\right), f_{v}^{-1}\left(y_{0}\right)\right)} \\
& +\frac{\operatorname{dil}\left(f^{-1}\right) d\left(f_{v}^{-1}\left(y_{0}\right), f_{v}^{-1}\left(\frac{1}{2^{n_{1}}}\right)\right)}{\sqrt{2} d\left(f_{v}^{-1}\left(x_{0}\right), f_{v}^{-1}\left(y_{0}\right)\right)} \\
\leq & \frac{1}{2^{\frac{1}{4}}} \operatorname{dil}\left(f^{-1}\right) .
\end{aligned}
$$

Here we used the equality (8) in the second and third lines, the equality (6) and the definition of the dilation in the fourth and fifth lines, and the inequality (7) in the last line. The inequality (9) implies that $\operatorname{dil}(f) \geq 2^{\frac{1}{8}}$ or $\operatorname{dil}\left(f^{-1}\right) \geq 2^{\frac{1}{8}}$. Thus we have

$$
d_{L}\left(Y_{u}, Y_{v}\right) \geq \frac{\log 2}{8} .
$$

This contradicts the inequality (5). Therefore we have, for any $n \in P_{u}$,

$$
f_{v} \circ f \circ f_{u}^{-1}\left(\frac{5}{2^{n+2}}\right) \notin\left\{\frac{1}{2^{m}}: m \in \mathbb{Z}_{>0}\right\} .
$$

By the same argument as above, we have, for any $n \in P_{u}$,

$$
f_{v} \circ f \circ f_{u}^{-1}\left(\frac{5}{2^{n+2}}\right) \notin\left\{\frac{3}{2^{m+1}}: m \in \mathbb{Z}_{>0}\right\} .
$$

Now we start to show the third step (iii). By the above two steps ( $i)$ and (ii), we have that, for any $n \in P_{u}$, there exists $p_{f}(n) \in \mathbb{Z}_{>0}$ such that

$$
f_{v} \circ f \circ f_{u}^{-1}\left(\frac{5}{2^{n+2}}\right)=\frac{5}{2^{p_{f}(n)+2}} .
$$

By the same argument as the first step (i), we can check that $p_{f}(n) \in P_{v}$, that is, $v_{p_{f}(n)}=1$. Also for the inverse function $f^{-1}$, we have that, for any $n \in P_{v}$, there exists $p_{f^{-1}}(n) \in P_{u}$ such that

$$
f_{u} \circ f^{-1} \circ f_{v}^{-1}\left(\frac{5}{2^{n+2}}\right)=\frac{5}{2^{p_{f^{-1}}(n)+2}} .
$$

Since $f$ is a bijection, the map $p_{f}$ is a bijection from $P_{u}$ to $P_{v}$ and $p_{f}^{-1}=p_{f^{-1}}$.

Now it suffices to show that $p_{f}(n)=n$ for all $n \in P_{u}$. We assume that there exists $l \in P_{u}$ such that $p_{f}(l) \neq l$.
Without loss of generality, we may assume $p_{f}(l)>l$. We first show that

$$
f_{u} \circ f^{-1} \circ f_{v}^{-1}\left(\frac{1}{2^{p_{f}(l)}}\right) \in\left(\frac{1}{2^{l}}, \frac{5}{2^{l+2}}\right) \cup\left(\frac{5}{2^{l+2}}, \frac{3}{2^{l+1}}\right) .
$$

To show this, it suffices to show that

$$
\frac{\sqrt{2}}{2^{l+2}}>d\left(f^{-1} \circ f_{v}^{-1}\left(\frac{1}{2^{p_{f}(l)}}\right), f_{u}^{-1}\left(\frac{5}{2^{l+2}}\right)\right),
$$

where the above inequality means that the point $f^{-1}$ 。 $f_{v}^{-1}\left(\frac{1}{2^{p_{f}(l)}}\right)$ belongs to one of two edges in the l-pulse crossing at the right angle. By $p_{f^{-1}} \circ p_{f}(l)=p_{f}^{-1} \circ p_{f}(l)=l$, we have

$$
\begin{aligned}
\frac{\sqrt{2} \operatorname{dil}\left(f^{-1}\right)}{2^{p_{f}(l)+2}} & =\operatorname{dil}\left(f^{-1}\right) d\left(f_{v}^{-1}\left(\frac{1}{2^{p_{f}(l)}}\right), f_{v}^{-1}\left(\frac{5}{2^{p_{f}(l)+2}}\right)\right) \\
& \geq d\left(f^{-1} \circ f_{v}^{-1}\left(\frac{1}{2^{p_{f}(l)}}\right), f^{-1} \circ f_{v}^{-1}\left(\frac{5}{2^{p_{f}(l)+2}}\right)\right) \\
& =d\left(f^{-1} \circ f_{v}^{-1}\left(\frac{1}{2^{p_{f}(l)}}\right), f_{u}^{-1}\left(\frac{5}{2^{p_{f}-1} \circ p_{f}(l)+2}\right)\right) . \\
& =d\left(f^{-1} \circ f_{v}^{-1}\left(\frac{1}{2^{p_{f}(l)}}\right), f_{u}^{-1}\left(\frac{5}{2^{l+2}}\right)\right) .
\end{aligned}
$$

Since we have dil $\left(f^{-1}\right) \leq 2^{\frac{1}{4}}$ (by the inequality (5)) and $p_{f}(l) \geq l+1$, it holds

$\frac{\sqrt{2}}{2^{l+2}}>\frac{\sqrt{2} d i l\left(f^{-1}\right)}{2^{p_{f}(l)+2}} \geq d\left(f^{-1} \circ f_{v}^{-1}\left(\frac{1}{2^{p_{f}(l)}}\right), f_{u}^{-1}\left(\frac{5}{2^{l+2}}\right)\right)$.

Thus we have shown (10).

By the continuity of $f$ and (10), there exists $\delta>0$ such that $\delta<\frac{1}{2^{p_{f}(l)+3}}$ and

$$
\begin{gathered}
f_{u} \circ f^{-1} \circ f_{v}^{-1}\left(\left[\frac{1}{2^{p_{f}(l)}}-\delta, \frac{1}{2^{p_{f}(l)}}+\delta\right]\right) \\
\subset\left(\frac{1}{2^{l}}, \frac{5}{2^{l+2}}\right) \cup\left(\frac{5}{2^{l+2}}, \frac{3}{2^{l+1}}\right) .
\end{gathered}
$$

Since the three points $f^{-1} \circ f_{v}^{-1}\left(\frac{1}{2^{p_{f}()}}-\delta\right), f^{-1} \circ$ $f_{v}^{-1}\left(\frac{1}{2^{p_{f}(l)}}\right)$ and $f^{-1} \circ f_{v}^{-1}\left(\frac{1}{2^{p_{f}(l)}}+\delta\right)$ are on the same line, we have

$$
\begin{aligned}
d( & \left.f^{-1} \circ f_{v}^{-1}\left(\frac{1}{2^{p_{f}(l)}}-\delta\right), f^{-1} \circ f_{v}^{-1}\left(\frac{1}{2^{p_{f}(l)}}+\delta\right)\right) \\
= & d\left(f^{-1} \circ f_{v}^{-1}\left(\frac{1}{2^{p_{f}(l)}}-\delta\right), f^{-1} \circ f_{v}^{-1}\left(\frac{1}{2^{p_{f}(l)}}\right)\right) \\
& +d\left(f^{-1} \circ f_{v}^{-1}\left(\frac{1}{2^{p_{f}(l)}}\right), f^{-1} \circ f_{v}^{-1}\left(\frac{1}{2^{p_{f}(l)}}+\delta\right)\right) .
\end{aligned}
$$


Thus the inclusion (10) and the equality (11) imply the following bound of the dilation off:

$$
\begin{aligned}
\operatorname{dil}\left(f^{-1}\right) & \\
\geq & \frac{d\left(f^{-1} \circ f_{v}^{-1}\left(\frac{1}{2^{p_{f}(l)}}-\delta\right), f^{-1} \circ f_{v}^{-1}\left(\frac{1}{2^{p_{f}(l)}}+\delta\right)\right)}{d\left(f_{v}^{-1}\left(\frac{1}{2^{p_{f}(l)}}-\delta\right), f_{v}^{-1}\left(\frac{1}{2^{p_{f}(l)}}+\delta\right)\right)} \\
= & \frac{d\left(f^{-1} \circ f_{v}^{-1}\left(\frac{1}{2^{p_{f}(l)}}-\delta\right), f^{-1} \circ f_{v}^{-1}\left(\frac{1}{2^{p_{f}(l)}}\right)\right)}{\sqrt{5} \delta} \\
& +\frac{d\left(f^{-1} \circ f_{v}^{-1}\left(\frac{1}{2^{p_{f}(l)}}\right), f^{-1} \circ f_{v}^{-1}\left(\frac{1}{2^{p_{f}(l)}}+\delta\right)\right)}{\sqrt{5} \delta} \\
\geq & \frac{d\left(f_{v}^{-1}\left(\frac{1}{2^{p_{f}(l)}}-\delta\right), f_{v}^{-1}\left(\frac{1}{2^{p_{f}(l)}}\right)\right)}{\sqrt{5} \delta \operatorname{dil}(f)} \\
= & \frac{d\left(f_{v}^{-1}\left(\frac{1}{2^{p_{f}(l)}}\right), f_{v}^{-1}\left(\frac{1}{2^{p_{f}(l)}}+\delta\right)\right)}{\sqrt{5} \delta \operatorname{dil}(f)} \\
\sqrt{2}+1 &
\end{aligned}
$$

Here we used the following equality in the third and fourth lines:

$$
\begin{aligned}
d & \left(f_{v}^{-1}\left(\frac{1}{2^{p_{f}(l)}}-\delta\right), f_{v}^{-1}\left(\frac{1}{2^{p_{f}(l)}}+\delta\right)\right) \\
& =\left(\left|\frac{1}{2^{p_{f}(l)}}-\delta-\left(\frac{1}{2^{p_{f}(l)}}+\delta\right)\right|^{2}+|\delta|^{2}\right)^{1 / 2} \\
& =\sqrt{5} \delta .
\end{aligned}
$$

Thus dil $(f) \geq\left(\frac{\sqrt{2}+1}{\sqrt{5}}\right)^{1 / 2}$ or $\operatorname{dil}\left(f^{-1}\right) \geq\left(\frac{\sqrt{2}+1}{\sqrt{5}}\right)^{1 / 2}$.

This contradicts the inequality (5). Therefore we have $p_{f}(n)=n$ for any $n \in P_{u}$.

We have completed all of the three steps. Setting $F(n)=$ $p_{f}(n)$, we have that the map $F: P_{u} \rightarrow P_{v}$ is a bijection such that $F(n)=n$ and this implies $P_{u}=P_{v}$. We have completed the proof.

We resume the proof of Theorem 2.

Proof of Theorem 2. By using Lemma 2, we know that $\left(\mathbb{Y}, d_{L}\right)$ is discrete. Since the cardinality of $\mathbb{Y}$ is continuum and $\mathbb{Y} \subset \mathcal{M}_{[0,1]}$, we have that $\left(\mathcal{M}_{[0,1]}, d_{L}\right)$ is not separable. We have completed the proof.

Remark 1. Theorem 2 says that $\mathcal{M}_{[0,1]}=\{X \in \mathcal{M}$ : $d([0,1], X)<\infty\}$ is not separable. By the proof of Theorem 2, moreover we know the following stronger result:

Let $B_{d_{L}}([0,1], \delta)$ denote the ball in $\mathcal{M}_{[0,1]}$ centered at $[0,1]$ with radius $\delta>0$ with respect to the Lipschitz distance $d_{L}$, that is,

$$
B_{d_{L}}([0,1], \delta)=\left\{X \in \mathcal{M}_{[0,1]}: d_{L}([0,1], X)<\delta\right\} .
$$

Then, for any $\delta>0, B([0,1], \delta)$ is not separable. In fact, let

$$
\begin{aligned}
J^{\epsilon}(n, 1)= & {\left[3 / 2^{n+1}, 1 / 2^{n-1}\right] \times\{0\} } \\
& \cup\left\{\left(x, \epsilon\left(3 / 2^{n+1}-x\right): 5 / 2^{n+2} \leq x \leq 3 / 2^{n+1}\right\}\right. \\
& \cup\left\{\left(x, \epsilon\left(x-1 / 2^{n}\right)\right): 1 / 2^{n} \leq x \leq 5 / 2^{n+2}\right\}, \\
J^{\epsilon}(n, 0)= & J(n, 0), \\
Y_{u}^{\epsilon}= & \{(0,0)\} \cup \bigcup_{n=1}^{\infty} J^{\epsilon}\left(n, u_{n}\right), u=\left(u_{n}\right)_{n \in \mathbb{Z}_{>0}} \in\{0,1\}^{\mathbb{Z}_{>0} .}
\end{aligned}
$$

Then, by the similar proof to that of Theorem 2, we obtain

(i) For every $\epsilon>0$, the set

$$
\mathbb{Y}^{\epsilon}=\left\{Y_{u}^{\epsilon}: u \in\{0,1\}^{\mathbb{Z}_{>0}}\right\} / \text { isometry }
$$

is discrete with cardinality of the continuum.

(ii) For every $\delta>0$, there exists $\epsilon>0$ such that $\mathbb{Y}^{\epsilon} \subset$ $B_{d_{L}}([0,1], \delta)$.

The statement (ii) implies that $B_{d_{L}}([0,1], \delta)$ is not separable for any $\delta>0$.

\section{Acknowledgment}

We would like to thank an anonymous referee for careful reading of our manuscript and pointing out Remark 1. The first author was supported by Grant-in-Aid for JSPS Fellows Number 261798 and DAAD PAJAKO Number 57059240

Received: 24 December 2014 Revised: 9 February 2015

Accepted: 18 February 2015

Published online: 31 March 2015

\section{References}

1. Burago D, Burago Y. and Ivanov. A course in metric geometry. Graduate Studies in Mathematics vol. 33. American Mathematical Society, Providence, Rl, (2001). Errata list available online at http://www.pdmi.ras. ru/ svivanov/papers/bbi-errata.pdf.

2. Gromov M. Metric Structures for Riemannian and Non-Riemannian Spaces. Birkhäuser Boston Inc, Boston, MA, 1999, Based on the 1981 French original [MR 85e:53051], With appendices by MKatz, P. Pansu, and S. Semmes, Translated from the French by Sean Michael Bates.

3. Sharapov S, Sharapov V. Dimensions of some generalized Cantor sets, preprint. http://classes.yale.edu/fractals/FracAndDim/cantorDims/ CantorDims.html.

4. Suzuki K. Convergence of continuous stochastic processes on compact metric spaces converging in the Lipschitz distance, preprint, arXiv:1412.0736. 